\title{
Prevalence of cervical pathogens in women with and without inflammatory changes on smear testing
}

\author{
Wanda L Parsons, Marshall Godwin, Carl Robbins, Roger Butler
}

Abstract

Objective-To assess correlation between nonspecific cervicitis, inflammation, or exudate on cervical smears tests and confirmed presence of known cervical pathogens.

Design-Investigation of women attending a family practice clinic for smear test by microbiological screening for Chlamydia. trachomatis, Mycoplasma hominis, Ureaplasma urealyticum, Trichomonas vaginalis, Candida species, group B streptococcus, Gardnerella vaginalis, and Neisseria gonorrhoeae.

Setting-Family practice teaching clinic in a university hospital.

Patients-411 women presenting for a smear test.

Main outcome measures-Prevalence of genital infections associated with presence or absence of inflammatory changes on cervical smear.

Results-Of the 132 women with inflammatory changes on cervical smear, $64(48 \%)$ had positive cultures. Of the $\mathbf{2 4 8}$ without inflammatory changes, $117(47 \%)$ had positive cultures. Subgroup analysis on individual organisms also showed no significant difference between the two groups.

Conclusion-Reports of inflammatory changes on cervical smear testing are a poor indicator of infection.

\section{Introduction}

Most laboratories reporting results of cervical smear tests comment on the possible presence of infection based on cytological criteria. Thus it is common to get a cervical smear report of non-specific cervicitis or inflammatory changes. The importance of these findings is unknown and no guidelines exist on appropriate management. Thus family doctors are often faced with the questions, should these women be recalled for cultures or treatment and are these women more likely to have positive cultures than women without inflammatory changes reported on smear testing?

This prompted one of us (WP) to recall for cervical cultures all women with reports of non-specific cervicitis on smear testing. This study determined that $40 \%$ of women with non-specific cervicitis on cervical smear tests had a positive culture for some organism. Kelly and Black in a study of a general practice found that $47 \%$ of women with inflammatory changes on cervical smear testing had a microbiologically proved infection. ${ }^{1}$ Wilson et al also found that inflammatory changes on cytology are often associated with infection. ${ }^{2}$

However, it is possible that just as many women with no inflammatory changes on smear testing would have positive cultures. Some studies have found a strong correlation between evidence of inflammation on smear testing and positive cultures for Chlamydia trachomatis, ${ }^{34}$ but others have found no correlation. ${ }^{5-9}$ The prevalence of $C$ trachomatis infections in women having cervical smear tests has also been examined. ${ }^{1011}$ We carried out cervical culture for a wide range of organisms in all women presenting for a smear test to determine the predictive value of inflammatory changes.

\section{Subjects and methods}

The family practice unit in St John's, Newfoundland, Canada, is a family practice teaching clinic located in a university hospital and associated with Memorial University. The clinic serves an urban population which is mostly middle class. All nonpregnant women seen at the unit for a smear test between November 1989 and May 1991 and who gave consent to be included in this study had microbiological investigation for $C$ trachomatis, Mycoplasma hominis, Ureaplasma urealyticum, group B streptococcus, Neisseria gonorrhoeae, Gardnerella vaginalis and Candida species.

Before the examination, the nature of the study was explained to the patient and verbal consent obtained. A questionnaire consisting of demographic and medical information was then administered by the doctor. The examination was then performed with the following protocol. The cervix was first visualised by the doctor using a standard clinical approach. Swabs were taken from the endocervix and initially a wet smear was taken to screen for Trichomonas vaginalis. A Gram stain was then done to screen for white cells, epithelial cells, yeast, and bacteria.

Swabs were then cultured on blood agar; chocolate agar; chocolate agar with vancomycin, colistin, and nystatin; and Columbia agar base with $5 \%$ human blood. The plates were screened for the presence of group B haemolytic streptococcus, Candida species, $G$ vaginalis, and $N$ gonorrhoeae. $C$ trachomatis and the mycoplasmas were tested for by inserting a calcium alginate swab into the endocervical canal and rotating the swab vigorously with gentle pressure for 5-10 seconds. Swabs were then placed in 2SP (sucrose phophate) transport medium containing no antimicrobial agents and immediately taken to the laboratory for testing for chlamydia and mycoplasma. ${ }^{12}$ Cervical smears were taken with a Cytobrush as well as standard bicornuate Papanicolaou smear sticks.

The cytologists who interpreted the smears were unaware that a study was being conducted. Women were considered to have evidence of inflammatory changes if the result of the smear test was reported as non-specific cervicitis, inflammation, or exudate. The cellular changes reported as cytological inflammation included enlarged nuclei deprived of nuclear structures, pyknosis or karyorrhesis, perinuclear halos, and cytoplasmic vacuolisation. The inflammatory patterns reported as non-specific cervicitis also included large numbers of neutrophils, increased exfoliation of keratinised superficial cells, increased numbers of parabasal cells, and generalised eosinophilia of the cells. ${ }^{13}$

\section{STATISTICAL ANALYSIS}

Results were analysed by $\chi^{2}$ ( $p$ values $\leqslant 0.05$ were considered significant), odds ratios, and Cohen's kappa $(\kappa)$. Positive and negative predictive values were also calculated. The study was approved by the human investigation committee.

\section{Results}

A total of 411 women had smear tests and cervical cultures completed. Thirty one of these were excluded 
because of technical problems, such as leaking cultures and overnight refrigeration. Of the remaining 380 women $132(35 \%)$ had inflammatory changes on smear testing and $248(65 \%)$ did not. No significant differences between the groups were identified (table I).

TABLE I-Demographic characteristics of women with and withou inflammatory changes on smear testing. Figures are numbers (percentages) unless stated otherwise

\begin{tabular}{lcc}
\hline & $\begin{array}{c}\text { With } \\
\text { inflammation } \\
(\mathrm{n}=132)\end{array}$ & $\begin{array}{c}\text { Without } \\
\text { inflammation } \\
(\mathrm{n}=248)\end{array}$ \\
\hline Mean age (years) & $32 \cdot 3$ & $33 \cdot 3$ \\
Nulliparous women & $56(42)$ & $122(49)$ \\
Mean parity of parous women & 2 & 2 \\
Contraception: & $44(33)$ & $106(43)$ \\
$\quad$ None & $40(30)$ & $73(29)$ \\
$\quad$ Oral & $48(36)$ & $69(28)$ \\
$\quad$ Other or unspecified & $12(9)$ & $20(8)$ \\
History of sexually transmitted disease & $7(5)$ & $27(11)$ \\
More than one sexual partner in past year & &
\end{tabular}

Table II shows the results of cultures in each group. Sixty four $(48 \%)$ women in the inflammation group had positive cultures compared with $117(47 \%)$ women in the non-inflammation group. The predictive values of both negative and positive results were not high enough to be of clinical value. With an odds ratio of 1.05 and $\kappa$ of 0.01 , the likelihood of having a positive culture with inflammatory changes was about the same as that without inflammatory changes. Subgroup analysis by individual organisms showed better negative predictive values ( $C$ trachomatis $98 \%$, group B streptococcus $94 \%$, candida $90 \%$ ) but the usefulness of this in the clinical situation is limited.

TABLE II-Results of culture in women with and without inflammatory changes on smear testing

\begin{tabular}{lccc}
\hline & Positive culture & Negative culture & Tota \\
\hline With inflammation & 64 & 68 & 132 \\
Without inflammation & 117 & 131 & 248 \\
\hline Total & 181 & 199 & 380 \\
\hline
\end{tabular}

Positive predictive value $48 \%$, negative predictive value $53 \%$.

Cohen's $\kappa=+0.01$

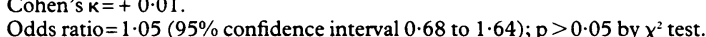

\section{Discussion}

Over a third of the women in this study had inflammatory changes on smear testing, and we found a high rate of positive cultures both in women with inflammatory changes and in those without inflammatory changes. The rate of infection was similar in the two groups for total positive cultures and for specific organisms. This suggests that inflammatory changes on smear testing are a poor predictor of cervical infection.

The prevalence of chlamydia infection $(1.5 \%)$ was much lower than that previously reported. ${ }^{10111415}$ There are several possible explanations for this. Firstly, our practice has a large number of women with stable monogamous relationships and the average age was 33 years, whereas chlamydia is seen primarily in younger women. ${ }^{15}$ Another possible explanation is that the cultures were not taken properly or were managed improperly before being processed by the laboratory. However, we had an explicit protocol for taking endocervical swabs and reviewed the protocol with all doctors before the beginning of the study. We also excluded women who were seen at the end of the day (after $4 \mathrm{pm}$ ) because of the possibility of their specimens being left overnight. The laboratory is in the same building as our clinic and specimens arrived at the laboratory within one hour of being obtained. Another possibility is a problem with methods used in the laboratory. However, the study was discussed at length with the microbiology laboratory and optimal methods of culture were used.

In summary, this study shows no correlation between the findings of non-specific cervicitis or other inflammatory changes on cervical smear tests and the presence of cervical pathogens. This suggests that a report of inflammatory changes cannot be used to reliably predict the presence of cervical infection and points to the importance of knowing the epidemiology of the practice in order to make appropriate decisions.

We thank Dr Susan King for her work on the preliminary pilot study, Dr Paul Fardy and Winston Brown of the microbiology laboratory, and the patients, staff, and physicians of the family practice unit.

1 Kelly BA, Black AS. The inflammatory cervical smear: a study in genera practice. Br f Gen Pract 1990;40:238-40.

2 Wilson JD, Robinson AJ, Kinghorn S, Hicks DA. Implications of inflammatory changes on cervical pathology. BMF 1990;300:638-40.

3 Kiviat NB, Paavonen JA, Brockway J, Critchlow CW, Brunham RC, Steven $\mathrm{CE}$, et al. Cytologic manifestations of cervical and vaginal infections. $¥ A M A$ 1985;253:989-1000.

4 Addiss DG, Vaughn ML, Hoclzhueter MA, Bakken LL, Davis JP. Selective screening for Chlamydia trachomatis infection in non-urban family planning clinics in Wisconsin. Fam Plann Perspect 1987;19:252-6.

5 Bertolino JG, Rangel JE, Blake RL, Silverstein D, Ingram E. Inflammation on the cervical Papanicolaou smear: the predictive value for infection in asymptomatic women. Fam Med 1992;24:447-52.

6 Dorman SA, Danos LM, Wilson DJ, Noller KL, Malkasian GD, Goellner JR et al. Detection of chlamydial cervicitis by Papanicolaou stained smears and culture. Am f Clin Pathol 1983;79:421-5.

7 Shafer MA, Chew KL, Kromhout LK, Beck A, Sweet L, Schachter J, et al. Chlamydial endocertical infections and cytologic findings in sexually active female adolescents. Am f Obstet Gynecol 1985;151:765-71.

8 Weismeier E, Rosenthal DL, Weideman S. Detection of chlamydial cervicitis with Papanicolaou-stained smears and cultures in a university studen population. I Reproduct Med 1987;32:251-3.

9 Geerling S, Nettum JA, Lindner LE, Miller SL, Dutton L, Wechter S. Sensitivity and specificity of the Papanicolaou-stained cervical smear in the diagnosis of Chlamydia trachomatis infection. Acta Cytologica 1985;29: $671-5$.

10 Smith JR, Murdock J, Carrington D, Frew CE, Dougall AJ, MacKinnon H, et al. Prevalence of Chlamydia trachomatis infection in women having et al. Prevalence of Chlamydia trachom

11 Masse R, Laperriere H, Rousseau H, Lefebvre J, Remis RS. Chlamydia trachomatis cervical infection: prevalence and determinants among women presenting for routine gynecologic examination. Can Med Assoc $\mathcal{F}$ 1991;145 $953-61$

12 Smith TF, Weed LA, Patterson GR, Segura JW. Recovery of chlamydia and genital mycoplasma transported in sucrose phosphate buffer and urease tes medium. Health Laboratory Science 1977;14:30-4.

13 Koss KG. Diagnostic cytology and its histopathologic bases. Philadelphia: Lippincott, 1992.

14 Bowie WR, Borrie-Hume CJ, Manzon LM, Fawcett A, Percival-Smith RKI, et al. Prevalence of Chlamydia trachomatis and Neisseria gonorrhoeae in two different populations of women. Can Med Assoc f 1981;124:1477-9.

15 Embil JA, Pereita LH. Prevalence of Chlamydia trachomatis and mycoplasmas in asymptomatic women. Can Med Assoc f 1985;138:34-5.

(Accepted 26 February 1992) 